\title{
Combining Stochastic and Scenario Model Predictive Control to Handle Target Vehicle Uncertainty in an Autonomous Driving Highway Scenario*
}

\author{
Tim Brüdigam ${ }^{1}$, Michael Olbrich ${ }^{1}$, Marion Leibold $^{1}$, and Dirk Wollherr ${ }^{1}$
}

\begin{abstract}
Autonomous vehicles face the challenge of providing safe transportation while efficiently maneuvering in an uncertain environment. Considering surrounding vehicles, two types of uncertainties occur: multiple future maneuvers are possible, and within these maneuvers the vehicle can vary from the predicted ideal maneuver path. Focusing on only one of these uncertainties can either lead to neglecting potential risks or result in overly conservative motion planning. Here, we suggest a Stochastic Model Predictive Control strategy that tackles the possibility of multiple future maneuvers of surrounding vehicles, while also considering uncertainty within the execution of these predicted maneuvers. The proposed control method is a combination of Scenario Model Predictive Control to cope with multiple predicted maneuvers of other vehicles, and Stochastic Model Predictive Control using chance-constraints to take into account vehicle deviations from the predicted maneuver trajectories of the respective maneuver. Adjustable risk parameters permit a violation of safety constraints up to a desired probability, allowing a trade-off between risk and performance. A simulation of a two-lane scenario demonstrates the effectiveness of our method.
\end{abstract}

\section{INTRODUCTION}

The past decade has seen an increasing interest in autonomous driving. Highway and urban traffic will remain at the center of attention for researchers working on autonomous vehicles, advancing safe and efficient transportation.

Autonomous driving requires dealing with environments that contain uncertainties, i.e., imperfect knowledge about current and future states of the controlled and surrounding vehicles. Among these uncertainties are sensor inaccuracy as well as vehicle maneuvers. Assuming that sensing will improve due to advances in technology, a major control challenge is to not necessarily only handle uncertainty in the state of the ego vehicle, i.e., the controlled vehicle, but effectively handle uncertainty in the predicted future motion of surrounding vehicles. There are two types of uncertainties when regarding other vehicles. First, vehicles can execute one of multiple possible maneuvers in the future, which is unknown to the controlled vehicle beforehand. Second, the precise execution of a future maneuver cannot be predicted with perfect accuracy, leading to trajectory uncertainty for each of the predicted possible maneuvers. Nevertheless,

\footnotetext{
*The authors gratefully acknowledge the financial support by the BMW Group

${ }^{1}$ Technical University of Munich, Department of Electrical and Computer Engineering, Chair of Automatic Control Engineering (LSR), 80333 Munich, Germany. Email: tim.bruedigametum. de
}

ensuring complete safety for all possible uncertainties drastically limits the performance of the autonomous vehicle. It is therefore necessary to find a reasonable trade-off between safety and efficiency.

In this paper we propose a combined Stochastic and Scenario Model Predictive Control (MPC) method, S+SC MPC, for trajectory planning that considers future motion of other vehicles, denoted as target vehicles in the following. In order to obtain an accurate prediction of target vehicle motion, we suggest predicting possible future target vehicle maneuvers, while at the same time including uncertainty in the maneuver execution. Adjustable risk parameters provide the possibility of weighting performance against safety.

MPC has proved to be effective for autonomous driving [1]-[3]. In MPC an optimal control problem for a finite horizon is repeatedly solved at each time step, while only the first input is applied to the system.

Recent advances in Stochastic Model Predictive Control (SMPC) and Scenario Model Predictive Control (SCMPC) are presented in [4], [5]. Multiple works focus on a trade-off between risk and conservativeness in autonomous driving. SMPC is used in [6] to find a trade-off between comfort and tracking quality in an autonomous driving example with fixed obstacles, while [7] focuses on lane keeping of semi-autonomous driving using the unscented transformation method to propagate the disturbance more precisely. In [8] an Interacting Multiple Model Kalman Filter is used to predict future positions of target vehicles. The predicted maneuver with the highest probability is then used to solve a chance-constrained MPC problem. In [9] a multipolicy decision-making algorithm is presented, which recursively chooses policies from a preselected set of possible policies, assuming that other traffic participants will perform the most likely of multiple possible policies in the future. SCMPC is applied in [10], [11] to plan lane change maneuvers for the controlled ego vehicle by sampling possible future target vehicle trajectories. Feasibility is ensured by assuming that it is always possible to abort the lane change maneuver.

However, to predict future target vehicle motion accurately, the number of samples necessary for SCMPC might be too high to be reasonable for an optimization problem. Yet, it is imprecise to predict target vehicle motion by only considering the most probable maneuver. Here, we tackle this issue by combining an SMPC method [12], [13] with an SCMPC approach [14], [15].

We use SCMPC to consider possible target vehicle maneu- 
vers. Samples of potential future target vehicle maneuvers are drawn according to the likelihood of each maneuver. A maneuver risk parameter is used to determine the necessary number of samples to keep the probability of a constraint violation below a specified level. If more samples are drawn, it is more likely that a maneuver that could cause a collision in the future is included in the prediction and then accounted for in the planning of the vehicle trajectory.

The SCMPC approach is combined with an SMPC method, using chance-constraints, to account for uncertainty in the predicted target vehicle trajectory for each of the predicted maneuvers. Assuming that the predicted target vehicle maneuver trajectory is not perfectly accurate, the SMPC method ensures that the probability of violating safety constraints due to this inaccuracy is below a specified value.

In summary, the contribution of this work is a combination of an SMPC method and SCMPC. This S+SC MPC approach accurately considers future behavior of target vehicles to plan trajectories and ensures that the risk of violating safety constraints remains below a desired level. Future target vehicle motion is accounted for by first predicting possible target vehicle maneuvers and then considering uncertainty within the target vehicle maneuver execution. This reduces both the necessary samples drawn for SCMPC and also the prediction error of the target vehicle motion for SMPC as only errors within a predicted maneuver need to be considered. The prediction error for SMPC does not need to cover maneuver uncertainty as this is accounted for by SCMPC. This yields an accurate target vehicle prediction while limiting the conservativeness of the estimation.

This paper begins with introducing the vehicle models and safety constraints in Sec. II. The S+SC MPC method is derived in Sec. III. The simulation, results, and a discussion are presented in Sec. IV, whereas concluding remarks follow in Sec. V.

\section{Modeling}

\section{A. Vehicle Models}

Two vehicle models are considered. The controlled vehicle will be denoted as the ego vehicle (EV) and surrounding vehicles are referred to as target vehicles (TVs). The TV model is used by the EV to predict future TV states. A linear, discrete point-mass model describes the dynamics of all vehicles. However, an uncertainty term is added to the TV model, which considers that the EV is unable to perfectly predict future TV states.

The dynamics of the EV are given by

$$
\boldsymbol{\xi}_{k+1}^{\mathrm{EV}}=A \boldsymbol{\xi}_{k}^{\mathrm{EV}}+B \mathbf{u}_{k}^{\mathrm{EV}}
$$

where $\boldsymbol{\xi}_{k}^{\mathrm{EV}}=\left[x_{k}^{\mathrm{EV}}, v_{x, k}^{\mathrm{EV}}, y_{k}^{\mathrm{EV}}, v_{y, k}^{\mathrm{EV}}\right]^{\top}$ and $\mathbf{u}_{k}^{\mathrm{EV}}=\left[u_{x, k}^{\mathrm{EV}}, u_{y, k}^{\mathrm{EV}}\right]^{\top}$ are the EV state and input at time step $k$, respectively, with the longitudinal position, velocity, and acceleration input $x_{k}^{\mathrm{EV}}, v_{x, k}^{\mathrm{EV}}$, and $u_{x, k}^{\mathrm{EV}}$ and the lateral position, velocity, and acceleration input $y_{k}^{\mathrm{EV}}, v_{y, k}^{\mathrm{EV}}$, and $u_{y, k}^{\mathrm{EV}}$. The system matrices are

$$
A=\left[\begin{array}{cccc}
1 & \Delta t & 0 & 0 \\
0 & 1 & 0 & 0 \\
0 & 0 & 1 & \Delta t \\
0 & 0 & 0 & 1
\end{array}\right], B=\left[\begin{array}{cc}
0.5(\Delta t)^{2} & 0 \\
\Delta t & 0 \\
0 & 0.5(\Delta t)^{2} \\
0 & \Delta t
\end{array}\right]
$$

with sampling time $\Delta t$.

For the TV we now consider uncertainty in future states using the continuous random variable $\mathbf{w}_{k}^{\mathrm{TV}}$ and the diagonal matrix $G=\operatorname{diag}\left(g_{1}, g_{2}, g_{3}, g_{4}\right)$. This results in the TV model

$$
\boldsymbol{\xi}_{k+1}^{\mathrm{TV}}=A \boldsymbol{\xi}_{k}^{\mathrm{TV}}+B \mathbf{u}_{k}^{\mathrm{TV}}+G \mathbf{w}_{k}^{\mathrm{TV}}
$$

with the TV state $\boldsymbol{\xi}_{k}^{\mathrm{TV}}=\left[x_{k}^{\mathrm{TV}}, v_{x, k}^{\mathrm{TV}}, y_{k}^{\mathrm{TV}}, v_{y, k}^{\mathrm{TV}}\right]^{\top}$ similar to (1) and the stabilizing feedback controller

$$
\begin{aligned}
\mathbf{u}_{k}^{\mathrm{TV}} & =K\left(\boldsymbol{\xi}_{k}^{\mathrm{TV}}-\boldsymbol{\xi}_{k, \mathrm{ref}}^{\mathrm{TV}}\right), \\
K & =\left[\begin{array}{cccc}
0 & k_{12} & 0 & 0 \\
0 & 0 & k_{21} & k_{22}
\end{array}\right] .
\end{aligned}
$$

In the following we will omit the index EV for the EV states and inputs.

Assumption 1. The vehicle models (1) and (3) describe exact $E V$ and $T V$ models, respectively.

Assumption 2. The TV model used by the EV to predict future TV states coincides with the actual TV model, i.e., the $E V$ has perfect knowledge of $A, B, G, K$, and $\boldsymbol{\xi}_{k \text {,ref }}^{\mathrm{TV}}$.

\section{B. Target Vehicle Prediction and Safety Constraint}

Two types of TV uncertainty are focused on for collision avoidance. First, possible TV maneuvers are predicted and then uncertainty within each predicted TV maneuver is considered as varying executions of the maneuvers are possible. This approach has the advantage that only small TV state deviations for each predicted maneuver need to be considered, instead of having to model and predict large future TV state deviations from the current TV state to account for all possible future TV maneuvers. The following will first address uncertainty in one maneuver and then focus on multiple possible maneuvers combined with maneuver execution uncertainty.

It is necessary to define a region around the TV that is considered unsafe for the EV, i.e, collision free motion is only ensured outside the restricted area. This is the safety constraint and is modeled by an ellipse around the TV given by

$$
d_{k}=\frac{\left(\Delta x_{k}\right)^{2}}{a^{2}}+\frac{\left(\Delta y_{k}\right)^{2}}{b^{2}}-1 \geq 0,
$$

which is used to prevent the EV from entering an ellipse with the TV as its center, as shown in Fig. 1. The safety constraint is satisfied if $d_{k} \geq 0$ and violated if $d_{k}<0$. The longitudinal and lateral distance between the EV and TV are

$$
\left[\begin{array}{c}
\Delta x_{k} \\
\Delta y_{k}
\end{array}\right]=\left[\begin{array}{l}
x_{k}^{\mathrm{EV}}-\hat{x}_{k}^{\mathrm{TV}} \\
y_{k}^{\mathrm{EV}}-\hat{y}_{k}^{\mathrm{TV}}
\end{array}\right]
$$

using the EV state and the TV state prediction $\hat{\boldsymbol{\xi}}_{k}^{\mathrm{TV}}=\left[\hat{x}_{k}^{\mathrm{TV}}, \hat{v}_{x, k}^{\mathrm{TV}}, \hat{y}_{k}^{\mathrm{TV}}, \hat{v}_{y, k}^{\mathrm{TV}}\right]^{\top}$. The ellipse semi-major and semi-minor axes are $a$ and $b$, respectively. 


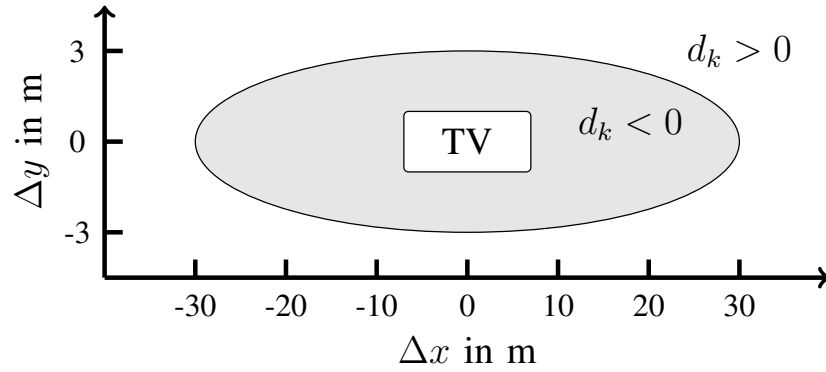

Fig. 1. Illustration of the safety constraint ellipse around the TV. The gray area is the constrained area. The TV is not drawn to scale.

Remark 1. An ellipse is used as the safety area around the TV to ensure that the constrained area is convex. Other convex shapes different than the proposed ellipse are possible [8].

Remark 2. The safety constraint ellipse around the TV can be chosen larger than the actual vehicle shape, i.e., slight violations of the safety constraint do not necessarily result in a collision.

After having addressed uncertainty in the predicted TV state, it is now considered that in addition the planned TV maneuver is uncertain.

Assumption 3. There are two lanes and two possible TV maneuvers: lane keeping $(L K)$ and lane changing $(L C)$ with the respective probabilities $p^{\mathrm{LK}}$ and $p^{\mathrm{LC}}=1-p^{\mathrm{LK}}$.

At every time step the EV predicts the TV maneuvers over a finite prediction horizon. Two TV maneuvers that start at the beginning of the prediction are considered. The TV is predicted to stay in its lane with probability $p=p^{\mathrm{LK}}$. With probability $p=p^{\mathrm{LC}}$ the EV expects that the TV will start a LC maneuver immediately, resulting in an altered lateral reference position $y_{k+1, \text { ref }}^{\mathrm{TV}}$. For the prediction horizon the TV is then expected to continue the initially chosen maneuver $y_{k+i \text {,ref }}^{\mathrm{TV}}=y_{k+1, \text { ref }}^{\mathrm{TV}}$, i.e., the lateral reference position can only change at the beginning and then remains constant throughout the prediction horizon. Only assuming immediate lane changes yields a more conservative estimation compared to considering possible lane changes at arbitrary points within the horizon. At the next time step, the EV starts a new TV maneuver prediction.

In other words, sampling the uniformly distributed random variable $p \in[0,1]$ and given the initial lateral reference position $y_{k, \text { ref }}^{\mathrm{TV}}$, the predicted future lateral TV reference positions $y_{k+i, \text { ref }}^{\mathrm{TV}}$ for the prediction horizon with $i \in 1, \ldots, N-1$ are

$$
y_{k+i, \text { ref }}^{\mathrm{TV}}= \begin{cases}y_{k, \text { ref }}^{\mathrm{TV}} & \text { if } \quad p \leq p^{\mathrm{LK}} \\ l_{\text {lane }}-y_{k, \text { ref }}^{\mathrm{TV}} & \text { else, }\end{cases}
$$

where $l_{\text {lane }}$ is the lane width. If for example the TV starts on the right lane, i.e. $y_{k \text {,ref }}^{\mathrm{TV}}=0$, and a sampled random variable $p \geq p^{\mathrm{LK}}$, i.e., a $\mathrm{LC}$ is sampled, the new lateral reference position $y_{k+i \text {,ref }}^{\mathrm{TV}}=l_{\text {lane }}$ is the left lane.

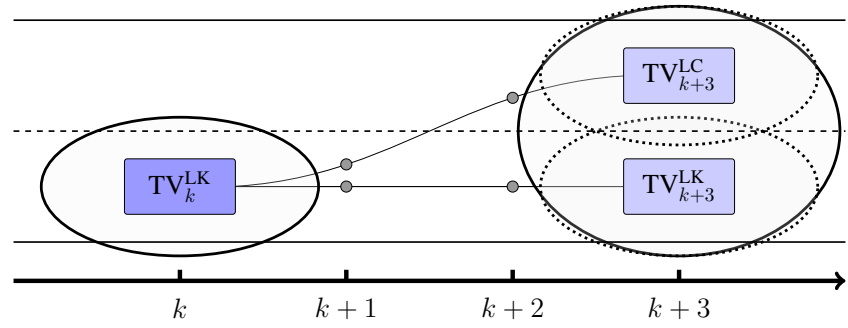

Fig. 2. Qualitative illustration of the combined safety constraint ellipse. The ellipse at time step $k$ only covers one maneuver (LK), while the larger ellipse at time step $k+3$ covers both individual ellipses for a LK and a LC maneuver, as both maneuvers are possible. Time steps $k+1$ and $k+2$ are only represented by gray dots, the ellipses are omitted here.

The safety constraint (5) is now adapted to hold for all possible maneuvers. Therefore, an ellipse is designed that covers the restricted region around the TV for both possible maneuvers, as displayed in Fig. 2. This yields

$$
\begin{aligned}
& \tilde{d}_{k}=\frac{\left(\Delta \tilde{x}_{k}\right)^{2}}{\tilde{a}^{2}}+\frac{\left(\Delta \tilde{y}_{k}\right)^{2}}{\tilde{b}^{2}}-1 \geq 0, \\
& \tilde{x}_{k}=x_{k}^{\mathrm{TV}, \mathrm{LC}}=x_{k}^{\mathrm{TV}, \mathrm{LK}}, \\
& \tilde{y}_{k}=\frac{y_{k}^{\mathrm{TV}, \mathrm{LC}}+y_{k}^{\mathrm{TV}, \mathrm{LK}}}{2},
\end{aligned}
$$

where $x_{k}^{\mathrm{TV}, \mathrm{LC}}, x_{k}^{\mathrm{TV}, \mathrm{LK}}$ denote the longitudinal position and $y_{k}^{\mathrm{TV}, \mathrm{LC}}, y_{k}^{\mathrm{TV}, \mathrm{LK}}$ represent the lateral position of the TV for both maneuvers. The result is an ellipse with center $\left(\tilde{x}_{k}, \tilde{y}_{k}\right)$ that contains the individual TV constraint ellipses of both maneuvers at the predicted time step $k$. The semi-major and semi-minor axes for the combined ellipse are

$$
\begin{aligned}
& \tilde{a}_{k}=a+\frac{2}{l_{\text {lane }}}\left(\tilde{b}_{k}-b\right), \\
& \tilde{b}_{k}=\left|0.5\left(y_{k}^{\mathrm{TV}, \mathrm{LC}}-y_{k}^{\mathrm{TV}, \mathrm{LK}}\right)\right|+b .
\end{aligned}
$$

Definition 1. The virtual state of the ellipse that combines individual maneuver ellipses is

$$
\begin{aligned}
\tilde{\boldsymbol{\xi}}_{k}^{\mathrm{TV}} & =\left[\tilde{x}_{k}^{\mathrm{TV}}, \tilde{v}_{x, k}^{\mathrm{TV}}, \tilde{y}_{k}^{\mathrm{TV}}, \tilde{v}_{y, k}^{\mathrm{TV}}\right]^{\top}, \\
& =\left[x_{k}^{\mathrm{TV}}, v_{x, k}^{\mathrm{TV}}, 0.5\left(y_{k}^{\mathrm{TV}, \mathrm{LC}}+y_{k}^{\mathrm{TV}, \mathrm{LK}}\right), v_{y, k}^{\mathrm{TV}}\right]^{\top}
\end{aligned}
$$

Remark 3. The combined ellipse is only an approximation and does not completely cover both individual ellipses.

Perfect coverage would require an area much larger than both individual ellipses, resulting in a highly restrictive safety constraint. The ellipses around the target vehicles must be chosen large enough so that the combined ellipse still covers the actual vehicles. The maximal neglected difference $d_{k}^{*}$ between the combined ellipse and the individual ellipses is obtained by solving

$$
d_{k}^{*}=\min d_{k} \text { s.t. } \tilde{d}_{k}=0 .
$$

This means the maximal violation can be found at the point where $\tilde{d}_{k}=0$, which is an admissible point using the combined ellipse, but it is within the constrained area of the individual ellipse. 


\section{Stochastic Model Predictive Control}

The overall aim is to plan safe ego vehicle trajectories by accounting for possible future target vehicle motion. A major challenge is to reduce the conservativeness of these planned trajectories, which can be tackled by exploiting the two sources of target vehicle uncertainty, i.e., multiple possible maneuvers and the specific execution of the respective maneuver.

MPC solves an optimization problem on a finite horizon. At each time step only the first input of the optimized input sequence is applied. This process is then repeated for the next time step. Assuming uncertainty in the TV state, we will first formulate an SMPC problem with chance-constraints for collision avoidance. Subsequently, we will derive an S+SC MPC method that takes into account the possibility of multiple TV maneuvers by using SCMPC, while still accounting for TV trajectory uncertainties.

Definition 2. Two risk parameters are considered. The maneuver risk parameter $\varepsilon_{\mathrm{m}}$ relates to the uncertainty due to multiple possible maneuvers, while the trajectory risk parameter $\varepsilon_{\mathrm{t}}$ accounts for uncertainty within the executed trajectory for a given maneuver.

\section{A. SMPC Formulation}

First, an SMPC problem is set up including a chanceconstraint. The parameter $\varepsilon_{\mathrm{t}}$ specifies the desired probability of constraint satisfaction, i.e., the chance-constraint may only be violated with probability $1-\varepsilon_{\mathrm{t}}$ at each predicted step.

The optimal control problem to be solved at each step is

$$
\begin{aligned}
\underset{\mathbf{U}}{\arg \min } & \sum_{k=0}^{N-1}\left(\left\|\Delta \boldsymbol{\xi}_{k}\right\|_{Q}^{2}+\left\|\mathbf{u}_{k}\right\|_{R}^{2}\right)+\left\|\Delta \boldsymbol{\xi}_{N}\right\|_{S}^{2}, \\
\text { s. t. } & \boldsymbol{\xi}_{k+1}=A \boldsymbol{\xi}_{k}+B \mathbf{u}_{k}, \quad k \in \mathbb{N}_{0}, \\
& \boldsymbol{\xi}_{k+j} \in \Xi, \quad j=1, \ldots, N-1, \\
& \boldsymbol{\xi}_{k+N} \in \Xi_{\mathrm{f}}, \\
& \mathbf{u}_{k+j} \in \mathcal{U}, \quad j=1, \ldots, N-1, \\
& \operatorname{Pr}\left(\boldsymbol{\xi}_{k+j} \in \Xi_{k+j}^{\text {safe }}\right) \geq \varepsilon_{\mathrm{t}}, \quad \varepsilon_{\mathrm{t}} \in[0.5,1]
\end{aligned}
$$

with the EV input $\mathbf{U}=\left(\mathbf{u}_{k}, \mathbf{u}_{k+1}, \ldots, \mathbf{u}_{k+N-1}\right)^{\top}$ and EV state $\boldsymbol{\xi}$, reference states $\boldsymbol{\xi}_{k \text {,ref }}$, time step $k$, prediction horizon $N$, and the sets of admissible states, terminal states, and inputs $\Xi, \Xi_{\mathrm{f}}$, and $\mathcal{U}$, respectively. The difference between the vehicle state and reference state is denoted by $\Delta \boldsymbol{\xi}_{k}=\boldsymbol{\xi}_{k}-\boldsymbol{\xi}_{k, \text { ref }}$ and the norm $\|\boldsymbol{\zeta}\|_{Z}^{2}$ is given by $\boldsymbol{\zeta}^{\top} Z \boldsymbol{\zeta}$. The matrices $Q, S \in \mathbb{R}^{4 \times 4}$ and $R \in \mathbb{R}^{2 \times 2}$ are weighting matrices. The set of safe states at each time step is denoted by $\Xi_{k+j}^{\mathrm{safe}}$.

The chance-constraint (13f) can be reformulated using (5) according to [8]. First, the error between the actual and the predicted TV state $\mathbf{e}_{k}^{\mathrm{TV}}=\boldsymbol{\xi}_{k}^{\mathrm{TV}}-\hat{\boldsymbol{\xi}}_{k}^{\mathrm{TV}}$ is defined with the predicted TV state $\hat{\boldsymbol{\xi}}_{k}^{\mathrm{TV}}$ and $\mathbf{e}_{k}^{\mathrm{TV}}=\left[e_{k, x}^{\mathrm{TV}}, e_{k, v_{x}}^{\mathrm{TV}}, e_{k, y}^{\mathrm{TV}}, e_{k, v_{y}}^{\mathrm{TV}}\right]^{\top}$. Given (6), linearizing (5) around the predicted TV state $\hat{\boldsymbol{\xi}}_{k}^{\text {TV }}$ yields

$$
d_{k}+\nabla d_{k} \mathbf{e}_{k}^{\mathrm{TV}} \geq 0
$$

where the gradient $\nabla d_{k}$ is

$$
\nabla d_{k}=\frac{\partial d_{k}}{\partial \hat{\boldsymbol{\xi}}_{k}^{\mathrm{TV}}}=\left[\frac{-2 \Delta x_{k}}{a^{2}}, \quad 0, \quad \frac{-2 \Delta y_{k}}{b^{2}}, 0\right] .
$$

Thus, the safety constraint (5) can be rewritten as $-\nabla d_{k} \mathbf{e}_{k}^{\mathrm{TV}} \leq d_{k}$ and therefore the chance-constraint (13f) is substituted by

$$
\operatorname{Pr}\left(-\nabla d_{k} \mathbf{e}_{k}^{\mathrm{TV}} \leq d_{k}\right) \geq \varepsilon_{\mathrm{t}},
$$

which can then be reformulated.

\section{B. Deterministic Reformulation of the SMPC Problem}

As the chance-constraint (16) cannot be applied directly, a transformation from the probabilistic to a deterministic problem is necessary. The constraint is thus split into a probabilistic equation and a deterministic inequality

$$
\begin{aligned}
& d_{k} \geq \gamma_{k}, \\
& \operatorname{Pr}\left(-\nabla d_{k} \mathbf{e}_{k}^{\mathrm{TV}} \leq \gamma_{k}\right)=\varepsilon_{\mathrm{t}} .
\end{aligned}
$$

Assumption 4. The distribution of the random variable $\mathrm{w}_{k}^{\mathrm{TV}}$ is known and normally distributed, i.e., $\mathbf{w}_{k}^{\mathrm{TV}} \sim \mathcal{N}\left(0, \Sigma_{w}^{\mathrm{TV}}\right)$ with the $T V$ covariance matrix $\Sigma_{w}^{\mathrm{TV}}=\operatorname{diag}\left(\left(\sigma_{x}^{\mathrm{TV}}\right)^{2},\left(\sigma_{v_{x}}^{\mathrm{TV}}\right)^{2},\left(\sigma_{y}^{\mathrm{TV}}\right)^{2},\left(\sigma_{v_{y}}^{\mathrm{TV}}\right)^{2}\right)$. The probability distribution of the prediction error for future TV states $\mathbf{e}_{k}^{\mathrm{TV}}$ is a known normal distribution $\mathbf{e}_{k}^{\mathrm{TV}} \sim \mathcal{N}\left(0, \Sigma_{k}^{e}\right)$ with the prediction error covariance matrix $\Sigma_{k}^{e}$.

Assumption 5. The initial TV state $\boldsymbol{\xi}_{0}^{\mathrm{TV}}$ is known with the initial error covariance matrix $\Sigma_{0}^{e}=0$.

Theorem 1. Let Assumption 1,2,4,5 hold. Then, the deterministic expression for the probabilistic chance-constraint (16) is

$$
\begin{aligned}
& d_{k} \geq \gamma_{k}, \\
& \gamma_{k}=\sqrt{2 \nabla d_{k} \Sigma_{k}^{e}\left(\nabla d_{k}\right)^{\top}} \operatorname{erf}^{-1}\left(2 \varepsilon_{\mathrm{t}}-1\right)
\end{aligned}
$$

with the inverse error function $\operatorname{erf}^{-1}($.$) and the prediction$ error covariance matrix $\Sigma_{k+1}^{e}=\Phi \Sigma_{k}^{e} \Phi^{\top}+G \Sigma_{w}^{\mathrm{TV}} G^{\top}$.

Proof. This proof is based on [8], which addresses EV state uncertainty. Introducing the matrix $\Phi=A+B K$, it follows that

$$
\boldsymbol{\xi}_{k+1}^{\mathrm{TV}}=\Phi \boldsymbol{\xi}_{k}^{\mathrm{TV}}-B K \boldsymbol{\xi}_{k, \text { ref }}^{\mathrm{TV}}+G \mathbf{w}_{k}^{\mathrm{TV}}
$$

Separating the target vehicle state $\boldsymbol{\xi}_{k}^{\text {TV }}$ into the sum of the estimated TV state $\hat{\boldsymbol{\xi}}_{k}^{\mathrm{TV}}$ and the prediction error $\mathbf{e}_{k}^{\mathrm{TV}}$ yields

$$
\begin{aligned}
\boldsymbol{\xi}_{k+1}^{\mathrm{TV}} & =\hat{\boldsymbol{\xi}}_{k+1}^{\mathrm{TV}}+\mathbf{e}_{k+1}^{\mathrm{TV}} \\
& =\left(\Phi \hat{\boldsymbol{\xi}}_{k}^{\mathrm{TV}}-B K \boldsymbol{\xi}_{k, \text { ref }}^{\mathrm{TV}}\right)+\left(\Phi \mathbf{e}_{k}^{\mathrm{TV}}+G \mathbf{w}_{k}^{\mathrm{TV}}\right) .
\end{aligned}
$$

Given Assumptions 4,5, the prediction error covariance matrix is

$$
\Sigma_{k+1}^{e}=\Phi \Sigma_{k}^{e} \Phi^{\top}+G \Sigma_{w}^{\mathrm{TV}} G^{\top} .
$$

This yields $-\nabla d_{k} \mathbf{e}_{k}^{\mathrm{TV}} \sim \mathcal{N}\left(0, \nabla d_{k} \Sigma_{k}^{e}\left(\nabla d_{k}\right)^{\top}\right)$. Using the quantile function for univariate normal distributions it follows that $\gamma_{k}=\sqrt{2 \nabla d_{k} \Sigma_{k}^{e}\left(\nabla d_{k}\right)^{\top}} \operatorname{erf}^{-1}\left(2 \varepsilon_{\mathrm{t}}-1\right)$. 
Remark 4. Here, TV state uncertainty is accounted for, which is different from [8] where only EV uncertainty is considered.

\section{Combined S+SC MPC Problem Formulation}

Using the constraint formulation (8), the SMPC problem (13) is now extended to handle multiple possible maneuvers while accounting for uncertainty within the execution of these maneuvers. An SCMPC approach is used in combination with the SMPC method of Sec. III-A, resulting in a combined approach. We will refer to this new method as S+SC MPC. At the beginning of each optimization problem we draw $K_{\varepsilon_{\mathrm{m}}}$ samples of $p$, evaluate the TV predictions using (7), and calculate the safety constraint according to (8), accounting for all sampled TV maneuvers. By introducing a tunable maneuver risk parameter $\varepsilon_{\mathrm{m}} \in(0,1)$, it is possible to define the necessary number of samples $K_{\varepsilon_{\mathrm{m}}} \in \mathbb{N}_{0}$ to ensure a desired safety level.

Theorem 2. Let Assumption 3 hold. Then, a sample size

$$
K_{\epsilon}>\log _{p^{\mathrm{LK}}}\left(\frac{\varepsilon_{\mathrm{m}}}{1-p^{\mathrm{LK}}}\right)
$$

satisfies that $\varepsilon_{\mathrm{m}}$ is greater than the probability $p^{*}$ of not having sampled a TV LC maneuver if an actual TV LC maneuver occurs.

Proof. The $K_{\varepsilon_{\mathrm{m}}}$ drawn samples $p^{(1)}, \ldots, p^{\left(K_{\varepsilon_{\mathrm{m}}}\right)}$ are independent and identically distributed. The probability that no sample includes a TV LC maneuver and simultaneously the TV actually performs a LC maneuver is therefore given by $p^{*}=p^{\mathrm{LC}}\left(p^{\mathrm{LK}}\right)^{K_{\varepsilon_{\mathrm{m}}}}=\left(1-p^{\mathrm{LK}}\right)\left(p^{\mathrm{LK}}\right)^{K_{\varepsilon_{\mathrm{m}}}}$. If $\varepsilon_{\mathrm{m}}>p^{*}$, then solving for $K_{\epsilon}$ yields (22). This ensures with a probability greater than $1-\varepsilon_{\mathrm{m}}$ that at least one LC maneuver had been sampled if a TV LC actually occurs at the next time step.

Remark 5. If the maneuver risk parameter is chosen to be $\varepsilon_{\mathrm{m}}>p^{\mathrm{LC}}$, no samples are necessary as a LC is less likely than the accepted probability $\varepsilon_{\mathrm{m}}$ of not having predicted a $L C$ if it actually occurs at the next time step.

In the following we will combine the scenario approach of Theorem 2 with the SMPC optimization problem of (13) and (18). First, the resulting optimization problem is stated. Then, the subsequent theorem will provide details for the computation of the chance-constraint.

The S+SC MPC method yields the optimization problem

$$
\begin{aligned}
\underset{\mathbf{U}}{\arg \min } & \sum_{k=0}^{N-1}\left(\left\|\Delta \boldsymbol{\xi}_{k}\right\|_{Q}^{2}+\left\|\mathbf{u}_{k}\right\|_{R}^{2}\right)+\left\|\Delta \boldsymbol{\xi}_{N}\right\|_{S}^{2}, \\
\text { s. t. } & \boldsymbol{\xi}_{k+1}=A \boldsymbol{\xi}_{k}+B \mathbf{u}_{k}, \quad k \in \mathbb{N}_{0}, \\
& \boldsymbol{\xi}_{k+j} \in \Xi, \quad j=1, \ldots, N-1, \\
& \boldsymbol{\xi}_{k+N} \in \Xi_{\mathrm{f}}, \\
& \mathbf{u}_{k+j} \in \mathcal{U}, \quad j=1, \ldots, N-1, \\
& \tilde{d}_{k} \geq \gamma_{k}, \\
& \gamma_{k}=\sqrt{2 \nabla \tilde{d}_{k} \tilde{\Sigma}_{k}^{e}\left(\nabla \tilde{d}_{k}\right)^{\top}} \operatorname{erf}^{-1}\left(2 \varepsilon_{\mathrm{t}}-1\right) .
\end{aligned}
$$

The framework of (23) is according to the SMPC formulation of (13). The chance-constraint in (23g), accounting for trajectory uncertainty, is updated so that it covers all TV maneuvers that need to be considered as determined by the the drawn samples $K_{\varepsilon_{\mathrm{m}}}$ according to the SCMPC approach. This results in an updated prediction error covariance matrix $\tilde{\Sigma}_{k}^{e}$ for the virtual ellipse combining individual maneuver ellipses.

Assumption 6. The prediction errors covariance matrix $\Sigma_{k}^{e}$ is equal for every maneuver and can be calculated using (21).

Theorem 3. Let Assumptions 1-6 hold. Given an adapted safety constraint ellipse due to drawing $K_{\varepsilon_{\mathrm{m}}}$ scenarios, the deterministic expression for the chance-constraint is

$$
\begin{aligned}
\tilde{d}_{k} & \geq \gamma_{k}, \\
\gamma_{k} & =\sqrt{2 \nabla \tilde{d}_{k} \tilde{\Sigma}_{k}^{e}\left(\nabla \tilde{d}_{k}\right)^{\top}} \operatorname{erf}^{-1}\left(2 \varepsilon_{\mathrm{t}}-1\right)
\end{aligned}
$$

with

$$
\begin{aligned}
& \tilde{\Sigma}_{k+1}^{e}=\Phi \tilde{\Sigma}_{k}^{e} \Phi^{\top}+G \tilde{\Sigma}_{w}^{\mathrm{TV}} G^{\top}, \\
& \tilde{\Sigma}_{w}^{\mathrm{TV}}=\operatorname{diag}\left(\left(\sigma_{x}^{\mathrm{TV}}\right)^{2},\left(\sigma_{v_{x}}^{\mathrm{TV}}\right)^{2}, 0.5\left(\sigma_{y}^{\mathrm{TV}}\right)^{2},\left(\sigma_{v_{y}}^{\mathrm{TV}}\right)^{2}\right) .
\end{aligned}
$$

Proof. Using the virtual state of the combined ellipse $\tilde{\boldsymbol{\xi}}_{k}^{\text {TV }}$ from Definition 1, we obtain

$$
\tilde{x}_{k}^{\mathrm{TV}}=x_{k}^{\mathrm{TV}} \sim \mathcal{N}\left(x_{k}^{\mathrm{TV}},\left(\sigma_{x}^{\mathrm{TV}}\right)^{2}\right)
$$

as the longitudinal position in both maneuvers, LK and LC, is equal. Similar to (26) it holds that

$$
\begin{aligned}
& \tilde{v}_{x, k}^{\mathrm{TV}} \sim \mathcal{N}\left(v_{x, k}^{\mathrm{TV}},\left(\sigma_{v_{x}}^{\mathrm{TV}}\right)^{2}\right), \\
& \tilde{v}_{y, k}^{\mathrm{TV}} \sim \mathcal{N}\left(v_{y, k}^{\mathrm{TV}},\left(\sigma_{v_{y}}^{\mathrm{TV}}\right)^{2}\right) .
\end{aligned}
$$

Assumption 6 with $\tilde{y}_{k}^{\mathrm{TV}}=0.5\left(y_{k}^{\mathrm{TV}, \mathrm{LK}}+y_{k}^{\mathrm{TV}, \mathrm{LC}}\right)$ yields

$$
\begin{aligned}
& 0.5 y_{k}^{\mathrm{TV}, \mathrm{LK}} \sim \mathcal{N}\left(0.5 y_{k}^{\mathrm{TV}, \mathrm{LK}}, 0.25\left(\sigma_{y}^{\mathrm{TV}}\right)^{2}\right), \\
& 0.5 y_{k}^{\mathrm{TV}, \mathrm{LC}} \sim \mathcal{N}\left(0.5 y_{k}^{\mathrm{TV}, \mathrm{LC}}, 0.25\left(\sigma_{y}^{\mathrm{TV}}\right)^{2}\right) .
\end{aligned}
$$

This leads to

$$
\tilde{y}_{k}^{\mathrm{TV}} \sim \mathcal{N}\left(0.5\left(y_{k}^{\mathrm{TV}, \mathrm{LK}}+y_{k}^{\mathrm{TV}, \mathrm{LC}}\right), 0.5\left(\sigma_{y}^{\mathrm{TV}}\right)^{2}\right) .
$$

It follows that $\tilde{w}_{k}^{\mathrm{TV}} \sim \mathcal{N}\left(0, \tilde{\Sigma}_{w}^{\mathrm{TV}}\right)$ with covariance matrix $\tilde{\Sigma}_{w}^{\mathrm{TV}}=\operatorname{diag}\left(\left(\sigma_{x}^{\mathrm{TV}}\right)^{2},\left(\sigma_{v_{x}}^{\mathrm{TV}}\right)^{2}, 0.5\left(\sigma_{y}^{\mathrm{TV}}\right)^{2},\left(\sigma_{v_{y}}^{\mathrm{TV}}\right)^{2}\right)$.

Splitting $\tilde{\boldsymbol{\xi}}_{k}^{\mathrm{TV}}$ into the estimated state of the combined ellipse $\hat{\tilde{\boldsymbol{\xi}}}_{k}^{\mathrm{TV}}$ and the prediction error $\tilde{\mathbf{e}}_{k}^{\mathrm{TV}} \sim \mathcal{N}\left(0, \tilde{\Sigma}_{k}^{e}\right)$ delivers

$$
\tilde{\Sigma}_{k+1}^{e}=\Phi \tilde{\Sigma}_{k}^{e} \Phi^{\top}+G \tilde{\Sigma}_{w}^{\mathrm{TV}} G^{\top} .
$$

With $\Delta \tilde{x}_{k}=\Delta x_{k}$ and $\Delta \tilde{y}_{k}=y_{k}-\hat{\tilde{y}}_{k}^{\mathrm{TV}}$ the gradient of $\tilde{d}_{k}$ is given by

$$
\nabla \tilde{d}_{k}=\frac{\partial \tilde{d}_{k}}{\partial \hat{\tilde{\xi}}_{k}^{\mathrm{TV}}}=\left[\begin{array}{llll}
\frac{-2 \Delta \tilde{x}_{k}}{\tilde{a}^{2}}, & 0, & \frac{-2 \Delta \tilde{y}_{k}}{\tilde{b}^{2}}, & 0
\end{array}\right],
$$


resulting in

$$
-\nabla \tilde{d}_{k} \tilde{\mathbf{e}}_{k}^{\mathrm{TV}} \sim \mathcal{N}\left(0, \nabla \tilde{d}_{k} \tilde{\Sigma}_{k}^{e}\left(\nabla \tilde{d}_{k}\right)^{\top}\right)
$$

which then yields the chance-constraint

$$
\gamma_{k}=\sqrt{2 \nabla \tilde{d}_{k} \tilde{\Sigma}_{k}^{e}\left(\nabla \tilde{d}_{k}\right)^{\top}} \operatorname{erf}^{-1}\left(2 \varepsilon_{\mathrm{t}}-1\right)
$$

using the quantile function for univariate normal distributions.

Remark 6. If a LC is not sampled, then $\tilde{y}_{k}^{\mathrm{TV}}=y_{k}^{\mathrm{TV}}$.

\section{Simulation STUdY}

Simulations to evaluate the presented method were carried out in MATLAB ${ }^{\circledR}$ using the MPC routine developed by [16] as the base for implementing our method. All quantities in this section are given in SI units unless stated otherwise.

A scenario with two lanes and one TV is assessed, utilizing the S+SC MPC method.

For all simulations we use a prediction horizon $N=20$, a time step $\Delta t=0.2$, and a total simulation time of $t_{\text {sim }}=10$. The EV and TV models are according to (1) and (3). The lane width is $l_{\text {lane }}=3.5$ and the EV follows the constraints

$$
\begin{aligned}
y_{k} & \in[-1.75,5.25], \\
u_{x, k} & \in[-5,5], \\
u_{y, k} & \in[-0.5,0.5], \\
\Delta u_{x, k+1} & \in[-1,1], \\
\Delta u_{y, k+1} & \in[-0.2,0.2]
\end{aligned}
$$

with $\Delta u_{x, k+1}=u_{x, k+1}-u_{x, k}$ for longitudinal and $\Delta u_{y, k+1}=u_{y, k+1}-u_{y, k}$ for lateral acceleration. The initial lateral position of all vehicles is the center of the vehicles' respective lanes. The reference $\boldsymbol{\xi}_{k \text {,ref }}=\left[0, v_{x, \text { ref }}, y_{k, \text { ref }}, 0\right]^{\top}$ is chosen so that the EV longitudinal velocity remains constant and it follows the lane which is closest to the EV center, i.e.,

$$
y_{k, \text { ref }}^{\mathrm{EV}}= \begin{cases}0 & \text { if } \\ l_{\text {lane }} & \text { else, }\end{cases}
$$

assuming a two-lane scenario where the lateral center of the right and left lane is 0 and $l_{\text {lane }}$, respectively.

The TV controller matrix values are $\left[k_{12}, k_{21}, k_{22}\right]=$ $[-1,-0.8,-2.2]$. The random variable $\mathbf{w}_{k}^{\mathrm{TV}} \sim \mathcal{N}\left(0, \Sigma_{w}^{\mathrm{TV}}\right)$ is a Gaussian distribution with covariance matrix $\Sigma_{w}^{\mathrm{TV}}=\operatorname{diag}(1,1,1,1)$ and the uncertainty matrix is given by $G=\operatorname{diag}(0.05,0.067,0.013,0.03)$, reflecting different TV uncertainty in lateral and longitudinal direction. The weighting matrices are $Q=\operatorname{diag}(0,2,0.5,0.1)$ and $R=\operatorname{diag}(1,0.1)$.

The length and width of all vehicles are assumed to be $6 \mathrm{~m}$ and $2 \mathrm{~m}$, respectively. Choosing $[a, b]=[30,3]$ the minimal allowed longitudinal and lateral distances between the EV and TV are $\left[\Delta x_{\min }, \Delta y_{\min }\right]=[24,1]$, assuming that the EV center is on the ellipse boundary for $d_{k}=0$. It follows from (12) that the combined ellipse considering two maneuvers has a maximal difference to the individual ellipses that is $d_{k}^{*}=-0.073$ which translates to a maximal longitudinal ellipse difference $\Delta \bar{x}=1.26$ for $y^{\mathrm{EV}}=y^{\mathrm{TV}}$ or lateral ellipse difference $\Delta \bar{y}=0.18$ for $x^{\mathrm{EV}}=x^{\mathrm{TV}}$, respectively.

\section{A. Recovery SMPC Problem Formulation}

As feasibility can become an issue for the MPC problem (23), we implement a recovery strategy that relies on an alternative MPC problem. The MPC problem (23) is altered by introducing a slack variable $\sigma_{k}$ to soften the chanceconstraint. This results in a recovery strategy MPC problem that is solved if the original MPC problem (23) becomes infeasible. For the next time step $k+1$ the original MPC (23) problem is solved again. The recovery problem is given by

$$
\begin{aligned}
\underset{\mathbf{U}, \sigma}{\arg \min } & \sum_{k=0}^{N-1}\left(\left\|\Delta \boldsymbol{\xi}_{k}\right\|_{\tilde{Q}}^{2}+\left\|\mathbf{u}_{k}\right\|_{\tilde{R}}^{2}+\lambda \sigma\right) \\
& +\left\|\Delta \boldsymbol{\xi}_{N}\right\|_{\tilde{S}}^{2} \\
\text { s. t. } & \boldsymbol{\xi}_{k+1}=A \boldsymbol{\xi}_{k}+B \mathbf{u}_{k}, \quad k \in \mathbb{N}_{0} \\
& \boldsymbol{\xi}_{k+j} \in \Xi, \quad j=1, \ldots, N-1 \\
& \boldsymbol{\xi}_{k+N} \in \Xi_{\mathrm{f}}, \\
& \mathbf{u}_{k+j} \in \mathcal{U}, \quad j=1, \ldots, N-1 \\
& \tilde{d}_{k} \geq \gamma_{k}-\sigma, \quad \sigma \geq 0, \\
& \gamma_{k}=\sqrt{\left.2 \nabla \tilde{d}_{k} \tilde{\Sigma}_{k}^{\mathrm{TV}} \nabla \tilde{d}_{k}\right)^{\top}} \operatorname{erf}^{-1}\left(2 \tilde{\varepsilon}_{\mathrm{t}}-1\right) .
\end{aligned}
$$

with the recovery strategy trajectory risk parameter $\tilde{\varepsilon}_{\mathrm{t}}$ and the positive slack variable $\sigma$ that transforms the deterministic chance-constraint (24) into a soft constraint. The weighting matrices $\tilde{Q}, \tilde{R}, \tilde{S}$ and the scalar slack variable $\lambda$ are chosen depending on the selected ratio between performance and conservativeness. Here, $\tilde{Q}=\operatorname{diag}(0,0.1,0.5,0.1), \tilde{R}=R$, and $\lambda=50$ are used.

Remark 7. The recovery strategy trajectory risk parameter $\tilde{\varepsilon}_{\mathrm{t}}$ can be chosen different from $\varepsilon_{\mathrm{t}}$ to put increased focus on constraint satisfaction.

\section{B. Two Vehicle Scenario with S+SC MPC Method}

In the simulated scenario the EV starts on the left lane and only one TV is considered, initially positioned in front of the $\mathrm{EV}$ on the right lane. The initial EV and TV states are given by $\boldsymbol{\xi}_{0}=[0,27,3.5,0]^{\top}$ and $\boldsymbol{\xi}_{0}^{\mathrm{TV}}=[29,24,0,0]^{\top}$ with the reference states $v_{x \text {,ref }}=27, y_{\text {ref }}=3.5, v_{x, \text { ref }}^{\mathrm{TV}}=24, y_{k, \text { ref }}^{\mathrm{TV}}=0$ for no TV LC, and $y_{k \text {,ref }}^{\mathrm{TV}}=3.5$ once a TV LC begins. The $\mathrm{EV}$ assumes that the probability of a TV LC maneuver is $p^{\mathrm{LC}}=0.1$ at every time step and that the maneuver will be executed completely once it has started. In the simulation two cases are distinguished: one scenario where the TV initiates a LC at $t_{\mathrm{LC}}=4 \mathrm{~s}$ and one where the TV keeps its lane. Different maneuver risk parameters $\varepsilon_{\mathrm{m}}$ are evaluated with $\varepsilon_{\mathrm{t}}=0.8$ and $\tilde{\varepsilon}_{\mathrm{t}}=0.995$. Each maneuver risk parameter and scenario is simulated 150 times. Positions of both vehicles at different time steps for a TV LC simulation are shown in Fig. 3(a) and 3(b), showing that a higher value $\varepsilon_{\mathrm{m}}$ leads to larger input values, Fig. 4(a), as the EV needs to account for an unexpected LC maneuver. If a LC maneuver is predicted, the EV reduces its velocity earlier, Fig. 4(b), allowing an increased distance to the TV compared to the case of no predicted LC, as shown in Fig. 4(c). In order to compare the 


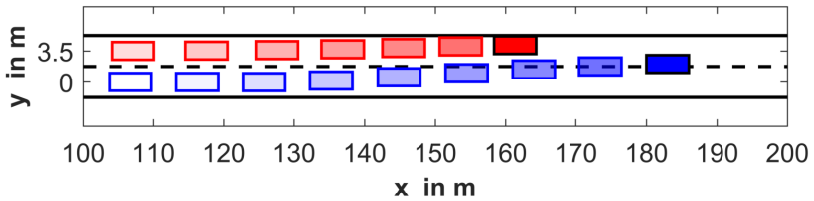

(a) Vehicles after $6.6 \mathrm{~s}$ at time step $k=33$ for $\varepsilon_{\mathrm{m}}=0.085$

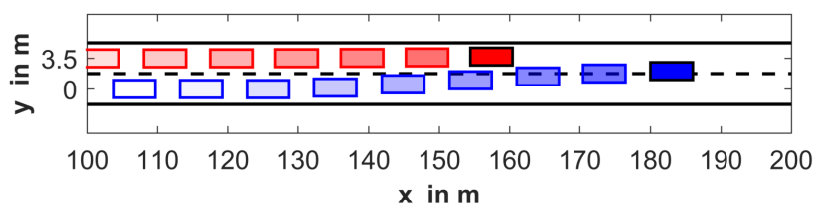

(b) Vehicles after $6.6 \mathrm{~s}$ at time step $k=33$ for $\varepsilon_{\mathrm{m}}=0.010$

Fig. 3. TV LC maneuver; red boxes represent the EV, blue boxes display the TV. Fading boxes show past states. Increasing space between past boxes represents acceleration. (a) The EV does not expect a TV LC maneuver and keeps a high velocity. Once the TV initiates a LC, the EV decelerates hard and moves to its left in order to increase the distance to the TV to regain $\tilde{d}_{k} \geq 0$. (b) The EV predicted a TV LC maneuver and is almost able to keep its longitudinal velocity and lateral position once the TV changes lanes while still satisfying $\tilde{d}_{k} \geq 0$.

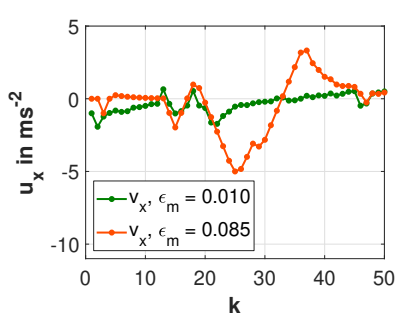

(a) Longitudinal acceleration of $\mathrm{EV}$

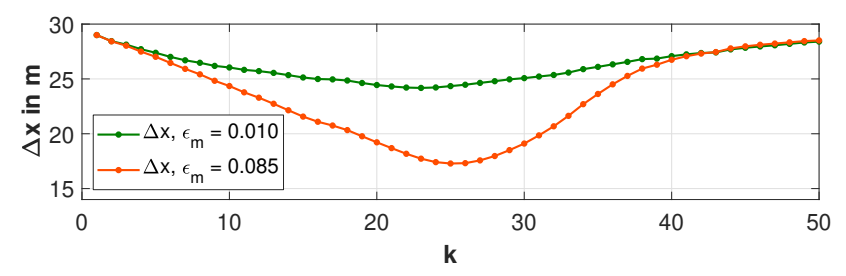

(c) Longitudinal distance between EV and TV

Fig. 4. TV LC maneuver with conservative (green) and aggressive (orange) maneuver risk parameter. (a) The longitudinal acceleration inputs are less smooth if the TV LC maneuver occurs unexpectedly due to a high $\varepsilon_{\mathrm{m}}$. (b) If a LC is predicted due to a low $\varepsilon_{\mathrm{m}}$, the EV decelerates earlier to be prepared for a possible TV LC. (c) If no LC is expected (high $\varepsilon_{\mathrm{m}}$ ), the longitudinal distance between EV and TV decreases, causing the EV to move to its left to keep a maximal possible distance to the TV.

results, similar to (13a) the total cost $J_{50}$ for all 50 steps is calculated by

$$
J_{50}=\sum_{k=0}^{49}\left(\left\|\Delta \boldsymbol{\xi}_{k}\right\|_{Q}^{2}+\left\|\mathbf{u}_{k}\right\|_{R}^{2}\right) .
$$

The averaged results of the simulations are depicted in Tab. I, where $d_{k, \text { min }}$ denotes the minimal value for $d_{k}$ in all simulated scenarios, which equals the maximal constraint violation.

If no TV LC occurs, a higher $\varepsilon_{\mathrm{m}}$, i.e., less maneuver samples drawn, leads to lower costs. However, if a TV LC maneuver occurs, for a high value $\varepsilon_{\mathrm{m}}$ the $\mathrm{EV}$ did not
TABLE I

COST AND CONSTRAINT VIOLATION FOR SMPC/SCMPC METHOD

\begin{tabular}{cccccc}
\hline & \multicolumn{2}{c}{ TV LC } & \multicolumn{2}{c}{ TV LK } \\
$\varepsilon_{\mathrm{m}}$ & $K_{\varepsilon_{\mathrm{m}}}$ & $J_{50}$ & $d_{k, \min }$ & $J_{50}$ & $d_{k, \min }$ \\
\hline 0.085 & 2 & 1700 & -0.151 & 39 & 0 \\
0.070 & 4 & 1484 & -0.104 & 197 & 0 \\
0.035 & 10 & 1092 & -0.017 & 583 & 0 \\
0.010 & 22 & 1014 & -0.016 & 640 & 0 \\
\hline
\end{tabular}

expect the LC maneuver and was unprepared. The EV needs to decelerate stronger, increasing the cost, and violates the safety constraint more than if a low value for $\varepsilon_{\mathrm{m}}$ is chosen. If $\varepsilon_{\mathrm{m}}$ is small, the sample size $K_{\varepsilon_{\mathrm{m}}}$ is larger and therefore the likelihood increases that a TV LC maneuver is predicted before it occurs.

\section{Discussion}

In the presented approach there are two risk parameters to be chosen, $\varepsilon_{\mathrm{t}}$ and $\varepsilon_{\mathrm{m}}$. By adjusting the maneuver risk parameter $\varepsilon_{\mathrm{m}}$ a trade-off is possible between performance and risk in the presence of multiple TV maneuvers. A lower value for $\varepsilon_{\mathrm{m}}$ leads to more samples considered, increasing the probability of having a larger restricted area. As handling uncertainty within the maneuver execution by only using SCMPC would require extensive modeling and sampling to cover all possible cases, a different approach, similar to SMPC with chance-constraints, is taken to ensure a specified level of constraint violation is not exceeded. This leads to the trajectory risk parameter $\varepsilon_{\mathrm{t}}$, which influences how much risk is accepted for the EV in the presence of TV trajectory uncertainty.

In the simulated scenarios the LC probability remains constant with the effect that a high number of samples $K_{\varepsilon_{\mathrm{m}}}$ is more likely to include a predicted LC maneuver than a small number $K_{\varepsilon_{\mathrm{m}}}$. One could argue that a maneuver risk parameter $\varepsilon_{\mathrm{m}}$ is not necessary, as in this scenario a TV LC could be considered if the focus is on safety, or a potential TV LC could be ignored if the main target is performance. However, the advantage of using the maneuver risk parameter $\varepsilon_{\mathrm{m}}$ lies in adaptability. In general, the probability of a TV LC maneuver is not constant, it changes depending on the situation and scenario: if a TV is faster than a vehicle in front, the LC probability is higher than in a scenario where the lane in front of the TV is empty. For each situation the EV updates the probability of a TV LC maneuver. With this belief, the EV then plans its trajectory according to the specified maneuver risk parameter $\varepsilon_{\mathrm{m}}$.

A relatively small sample size $K_{\varepsilon_{\mathrm{m}}}$, given by the maneuver risk parameter $\varepsilon_{\mathrm{m}}$, can be used to first evaluate if a TV LC maneuver needs to be considered. Then, the trajectory risk parameter $\varepsilon_{\mathrm{t}}$ specifies how conservative the planned EV motion is, given the TV maneuvers to be considered as determined in the previous step. A positive effect on safety can be achieved by increasing the trajectory risk parameter $\varepsilon_{\mathrm{t}}$ or decreasing the maneuver risk parameter $\varepsilon_{\mathrm{m}}$.

In the simulation results it is visible that a safety constraint violation does not necessarily lead to a collision as the safety 
ellipse is chosen larger than the TV. For $\varepsilon_{\mathrm{m}}=0.085$ the maximal constraint violation simulated was $d_{k, \min }=-0.151$ which translates to a minimal distance between the vehicle bodies of $\Delta x_{\min }^{*}=21.6 \mathrm{~m}$ in longitudinal direction for $y^{\mathrm{EV}}=y^{\mathrm{TV}}$, or $\Delta y_{\min }^{*}=0.7 \mathrm{~m}$ in lateral direction for $x^{\mathrm{EV}}=x^{\mathrm{TV}}$. These values indicate that no actual collision occurred even though the safety constraint was violated. This also means that the safety ellipse parameters need to be considered when aiming at improving performance.

In this work the focus lies on designing a method to combine SMPC and SCMPC that can be used for autonomous driving. However, the accuracy of the vehicle models needs to be increased. This could be done by including kinematic or dynamic bicycle models [17], which provide a more precise approximation of actual vehicle behavior [18].

\section{CONCLUSION}

We presented a combined method of SMPC and SCMPC. This S+SC MPC approach is suitable to plan vehicle trajectories that satisfy a specified safety level in the presence of multiple possible maneuvers of other vehicles, including uncertainty within the target vehicle maneuver execution. With this approach a trade-off between risk and performance is possible by choosing risk parameters according to a desired risk level. Uncertainties arising from the two sources, possible target vehicle maneuvers and maneuver execution, can be handled individually, allowing for less conservative ego vehicle motion planning. Future work includes applying the proposed method to more accurate vehicle models, as well as increasing the number of target vehicles and target vehicle maneuvers that are considered by the ego vehicle.

\section{REFERENCES}

[1] C. Katrakazas, M. Quddus, W.-H. Chen, and L. Deka, "Real-time motion planning methods for autonomous on-road driving: State-ofthe-art and future research directions," Transportation Research Part C: Emerging Technologies, vol. 60, pp. 416-442, 2015.

[2] J. Levinson, J. Askeland, J. Becker, J. Dolson, D. Held, S. Kammel, J. Z. Kolter, D. Langer, O. Pink, V. Pratt, M. Sokolsky, G. Stanek, D. Stavens, A. Teichman, M. Werling, and S. Thrun, "Towards fully autonomous driving: Systems and algorithms," in 2011 IEEE Intelligent Vehicles Symposium (IV), Baden-Baden, Germany, June 2011, pp. 163-168.

[3] A. Gray, Y. Gao, T. Lin, J. K. Hedrick, H. E. Tseng, and F. Borrelli, "Predictive control for agile semi-autonomous ground vehicles using motion primitives," in 2012 American Control Conference (ACC), Montreal, Canada, June 2012, pp. 4239-4244.

[4] M. Farina, L. Giulioni, and R. Scattolini, "Stochastic linear model predictive control with chance constraints a review," Journal of Process Control, vol. 44, no. Supplement C, pp. 53 - 67, 2016.

[5] A. Mesbah, "Stochastic model predictive control: An overview and perspectives for future research," IEEE Control Systems, vol. 36, no. 6, pp. 30-44, Dec 2016.

[6] D. Lenz, T. Kessler, and A. Knoll, "Stochastic model predictive controller with chance constraints for comfortable and safe driving behavior of autonomous vehicles," in 2015 IEEE Intelligent Vehicles Symposium (IV), Seoul, South Korea, June 2015, pp. 292-297.

[7] C. Liu, A. Gray, C. Lee, J. K. Hedrick, and J. Pan, "Nonlinear stochastic predictive control with unscented transformation for semiautonomous vehicles," in 2014 American Control Conference, Portland, USA, June 2014, pp. 5574-5579.

[8] A. Carvalho, Y. Gao, S. Lefevre, and F. Borrelli, "Stochastic predictive control of autonomous vehicles in uncertain environments," in $12 \mathrm{th}$ International Symposium on Advanced Vehicle Control, Tokyo, Japan, 2014.
[9] A. G. Cunningham, E. Galceran, R. M. Eustice, and E. Olson, "MPDM: Multipolicy decision-making in dynamic, uncertain environments for autonomous driving," in 2015 IEEE International Conference on Robotics and Automation (ICRA), Seattle, USA, May 2015, pp. 1670-1677.

[10] G. Schildbach and F. Borrelli, "Scenario model predictive control for lane change assistance on highways," in 2015 IEEE Intelligent Vehicles Symposium (IV), Seoul, South Korea, June 2015, pp. 611-616.

[11] G. Cesari, G. Schildbach, A. Carvalho, and F. Borrelli, "Scenario model predictive control for lane change assistance and autonomous driving on highways," IEEE Intelligent Transportation Systems Magazine, vol. 9, no. 3, pp. 23-35, Fall 2017.

[12] A. T. Schwarm and M. Nikolaou, "Chance-constrained model predictive control," AIChE Journal, vol. 45, no. 8, pp. 1743-1752, 1999.

[13] B. Kouvaritakis, M. Cannon, S. V. Rakovic, and Q. Cheng, "Explicit use of probabilistic distributions in linear predictive control," Automatica, vol. 46, no. 10, pp. 1719 - 1724, 2010.

[14] G. Schildbach, L. Fagiano, C. Frei, and M. Morari, "The scenario approach for stochastic model predictive control with bounds on closed-loop constraint violations," Automatica, vol. 50, no. 12, pp. 3009 - 3018, 2014

[15] M. C. Campi and S. Garatti, "A sampling-and-discarding approach tochance-constrained optimization: Feasibility and optimality," Journal of Optimization Theory and Applications, vol. 148, no. 2, pp. 257-280, Feb 2011.

[16] L. Grüne and J. Pannek, Nonlinear Model Predictive Control. London: Springer-Verlag, 2017.

[17] R. Rajamani, Vehicle Dynamics and Control, ser. Mechanical Engineering Series. Springer US, 2005.

[18] J. Kong, M. Pfeiffer, G. Schildbach, and F. Borrelli, "Kinematic and dynamic vehicle models for autonomous driving control design," in 2015 IEEE Intelligent Vehicles Symposium (IV), Seoul, South Korea, June 2015, pp. 1094-1099. 